
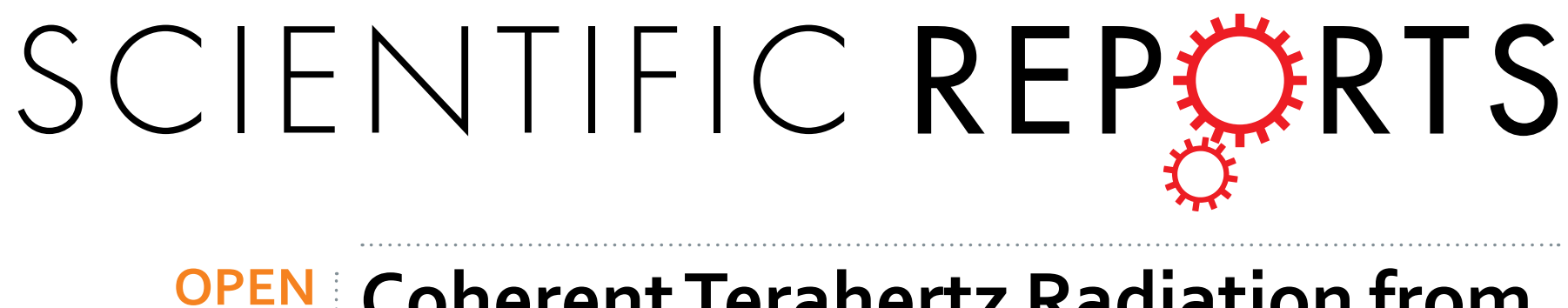

\title{
Coherent Terahertz Radiation from Multiple Electron Beams Excitation within a Plasmonic Crystal-like
}

Received: 21 June 2016

Accepted: 15 December 2016

Published: 23 January 2017

\section{structure}

\author{
Yaxin Zhang ${ }^{1,2}$, Yucong Zhou ${ }^{1}$, Yin Gang ${ }^{1}$, Guili Jiang ${ }^{1}$ \& Ziqiang Yang ${ }^{1}$
}

Coherent terahertz radiation from multiple electron beams excitation within a plasmonic crystal-like structure (a three-dimensional holes array) which is composed of multiple stacked layers with $3 \times 3$ subwavelength holes array has been proposed in this paper. It has been found that in the structure the electromagnetic fields in each hole can be coupled with one another to construct a composite mode with strong field intensity. Therefore, the multiple electron beams injection can excite and efficiently interact with such mode. Meanwhile, the coupling among the electron beams is taken place during the interaction so that a very strong coherent terahertz radiation with high electron conversion efficiency can be generated. Furthermore, due to the coupling, the starting current density of this mechanism is much lower than that of traditional electron beam-driven terahertz sources. This multi-beam radiation system may provide a favorable way to combine photonics structure with electronics excitation to generate middle, high power terahertz radiation.

Terahertz (THz) frequency band has been demonstrated of great interest for applications in biomedical imaging, communication, and security checking as the electromagnetic (EM) waves have various physical properties merging photonics and electronics ${ }^{1-3}$. The exploitation of these applications progresses slowly at present for the lack of powerful and compact $\mathrm{THz}$ sources. THz waves are usually obtained by optical devices ${ }^{4-6}$, solid state electron devices (SSEDs) $)^{7-9}$ and vacuum electron devices (VEDs) ${ }^{10-12}$. Although many progresses have been achieved, the output power level so far is still hard to keep up with demand of the applications ${ }^{11,12}$. Compared to the optical devices and SSEDs, the VEDs are more favorable to achieve high power since the electron transport medium is vacuum, which is a "perfect material" for high power devices ${ }^{12}$. Among the VEDs, the linear electron beam (e-beam)-driven sources have been actively investigated in the $\mathrm{THz}$ region ${ }^{13-19}$. However, for these radiation sources, with increased working frequency, higher starting current density is required and lower radiated power obtained $^{17}$. Therefore, many efforts in the linear e-beam-driven sources are concentrated on the design of effective interaction structures as well as on the interaction mechanism so as to improve the radiation intensity and to lower the starting current density.

Multi-beam sources are highly promising candidates to reduce the starting current density and to improve the radiated power. Therefore, in the earlier days, multi-beams have been applied in the microwave sources such as klystrons and free-electron lasers (FELs) ${ }^{20-23}$. Recently, there are some papers concentrating on multiple beamwave interaction in the $\mathrm{THz}$ region. Such as refs $24-27$ suggested two sheet e-beams interaction with mimicking surface plasmons (MSPs); ref. 28 proposed two sheet e-beams interaction within a composite sandwich structure; ref. 29 reported three- and six-beam folded waveguide traveling-wave tube (TWT) operating at fundamental mode; refs 30-34 presented two and three e-beams interaction with higher order mode; and ref. 35 theoretically investigated wave coupling in multi-beam TWTs. Although strong efforts have been made and some progress has been achieved in this aspect, an effective mechanism for the multi-beam interaction still seems to be a problem, especially for the case of more than 5 e-beams. Nowadays, the successful exploration of the plasmonic crystal $^{36-38}$ may lead to a bright perspective of multi-beam $\mathrm{THz}$ radiation sources.

${ }^{1}$ Terahertz Science and Technology Research Center, School of Physical Electronics, University of Electronic Science and Technology of China, Chengdu 610054, China. ${ }^{2}$ National Key Laboratory of Application Specific Integrated Circuit, Hebei Semiconductor Research Institute, China. Correspondence and requests for materials should be addressed toY.Z. (email: zhangyaxin@uestc.edu.cn) 


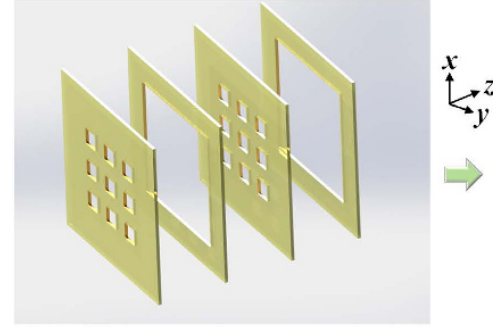

(a)

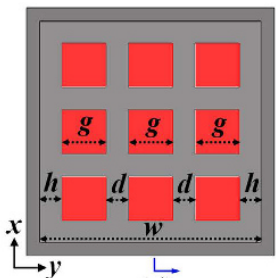

$(\overleftrightarrow{d)}$

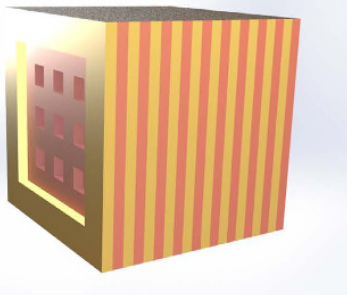

(b)

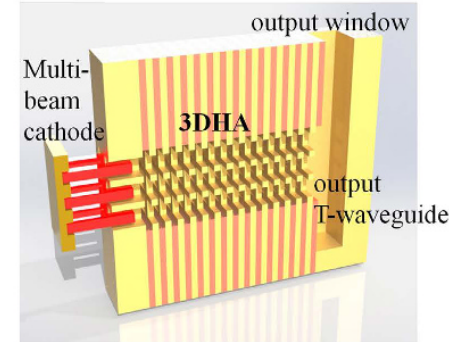

(c)

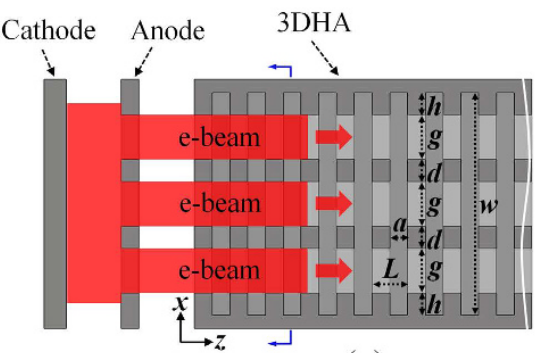

(e)

Figure 1. Sketch map of the 3DHA structure. (a) Two periodic units of the 3DHA. (b) 3DHA consist of several periodic units. (c) Metallic modal with multi-beam trajectories (cutaway view) of this source. (d,e) Cross section and longitudinal section of the 3DHA, respectively.

In ref. 16, we have proposed the interaction between a square-shaped e-beam with guiding wave mode in multiple stacked layers with single sub-wavelength holes (MSLS). The most important is that the MSLS can be can be integrated to construct a plasmonic crystal-like structure that can support multi-beam interaction. Moreover, the coupling of the electromagnetic fields may bring an enhancement of the interaction. Therefore, in this paper, we have proposed an efficient way to generate $\mathrm{THz}$ wave from the multi-beam interaction in such plasmonic crystal-like structure.

The plasmonic crystal that made of three-dimensional hole-array layers (3DHA) can support MSP waves which give the possibility of electron beam-MSP interaction and can also provide holes array to act as multi-beam channels. Therefore, multi-beams (9-beams) excitation within such structure which could lead to middle and high output power $\mathrm{THz}$ radiation has been studied in detail. The results show that such radiation source system leads to a significant improvement with higher efficiency and lower starting current density than traditional e-beam-driven sources.

\section{Results}

The model and electromagnetic coupling mode. The 3DHA structure is made of multiple stacked layers with $3 \times 3$ subwavelength holes array. Figure 1(a) illustrates two periodic units, and each periodic unit is composed of two different hole-layers. One of them is with a large hole and the other is with uniformly distributed $3 \times 3$ smaller holes array. Each layer can be fabricated separately with metal such as copper. Then, several periodic units are assembled to form the 3DHA structure (Fig. 1(b)). Figure 1(c) demonstrates a 3-D view of the whole design of this 3DHA THz source. A direct-current (DC) e-beam is extracted from the cathode to the anode on which centered $3 \times 3$ holes array is constructed. Some electrons are intercepted by the anode while the others pass through the $3 \times 3$ holes array on it to form $3 \times 3$ multi-beam array and then pass through the 3 DHA as shown in Fig. 1(c,e). The cross-sectional and longitudinal views of this structure with multi-beam trajectories and dimensional parameters are shown in Fig. $1(\mathrm{~d}, \mathrm{e})$, respectively. It can be found that each hole of the 3DHA acts as an e-beam channel where the injected e-beam passes through and interacts with the MSP wave in the structure.

As we know, for the coherent radiation from the beam-wave interaction, the field intensity and distribution in the structure are key factors. Firstly, the EM field distribution in the 3DHA has been investigated by applying the finite-integral-technique (FIT) eigenmode solver in CST Microwave Studio ${ }^{39}$.

Figure 2 demonstrates that the field distributions in different hole-array structures, where the boundary condition is perfect electric conductor (PEC). For the 1-hole structure in Fig. 2(a), the field distribution is regular around the structure. With the number of holes increasing, the field distribution has been changed as shown in Fig. 2(b-d). Due to the wave coupling among the holes, the distortion of the field takes place. On the other hand, in the longitudinal section, the contour map of the field distribution demonstrates that the mode in the 3DHA has the same EM characteristics of surface plasmons. However, due to the coupling in the center, the mode is not an evanescent wave so that this mode is just a mimicking surface plasmon wave.

The dispersion relation further shows the EM characteristics of this mode. It can be found from Fig. 3 that with the number of holes decreasing, the upper and cutoff frequencies increase and the dispersion passband becomes narrower. It should also be noted that, since the wave can be coupled through the holes array, 1st spatial harmonics of the dispersion curves in $3 \times 3$ hole-array structures are backward waves while it is forward wave for the 1-hole structure. 
(a)

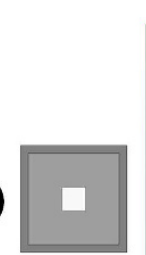

(b)

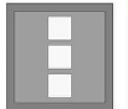

(c)

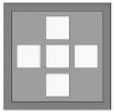

(d)
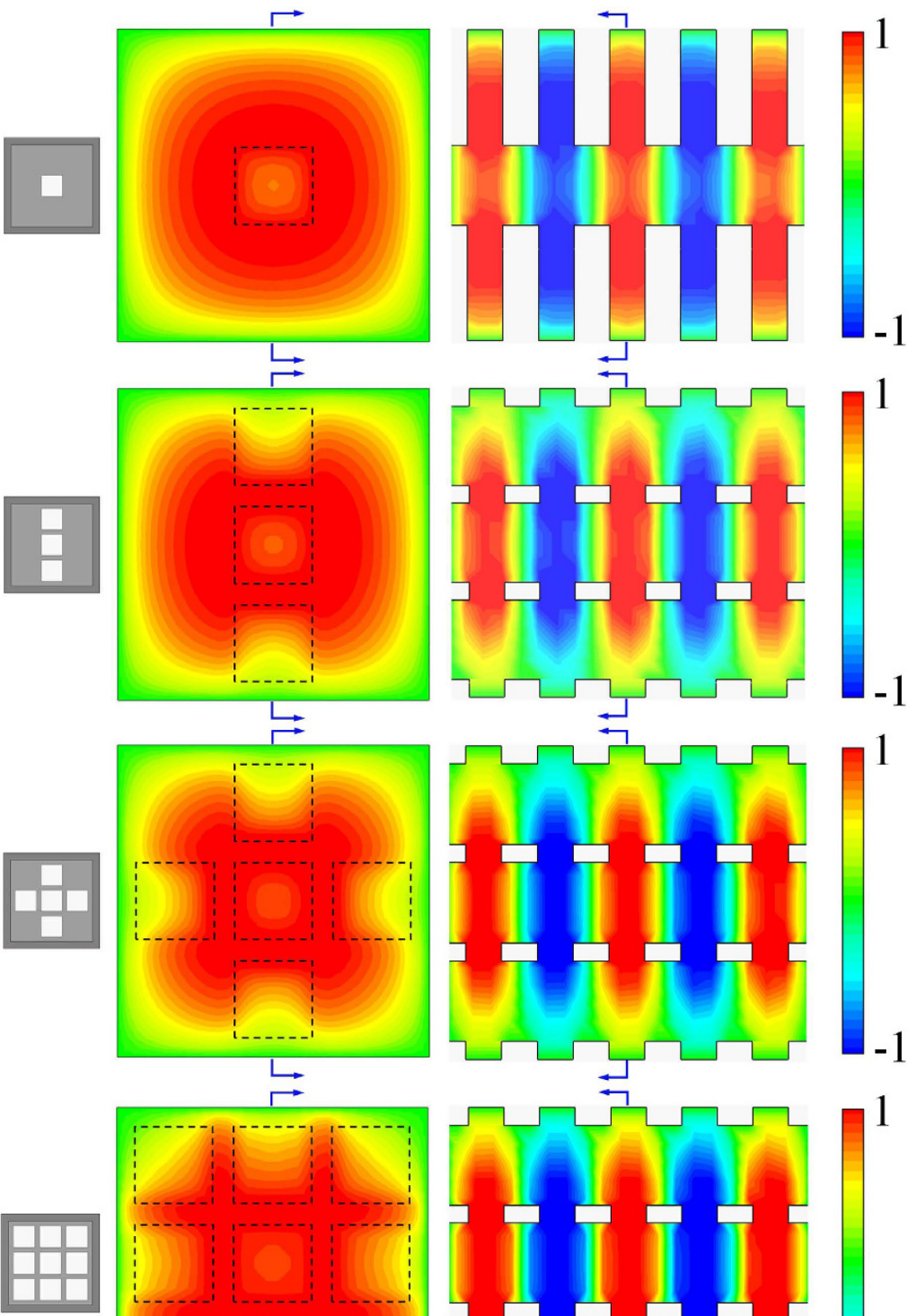

1
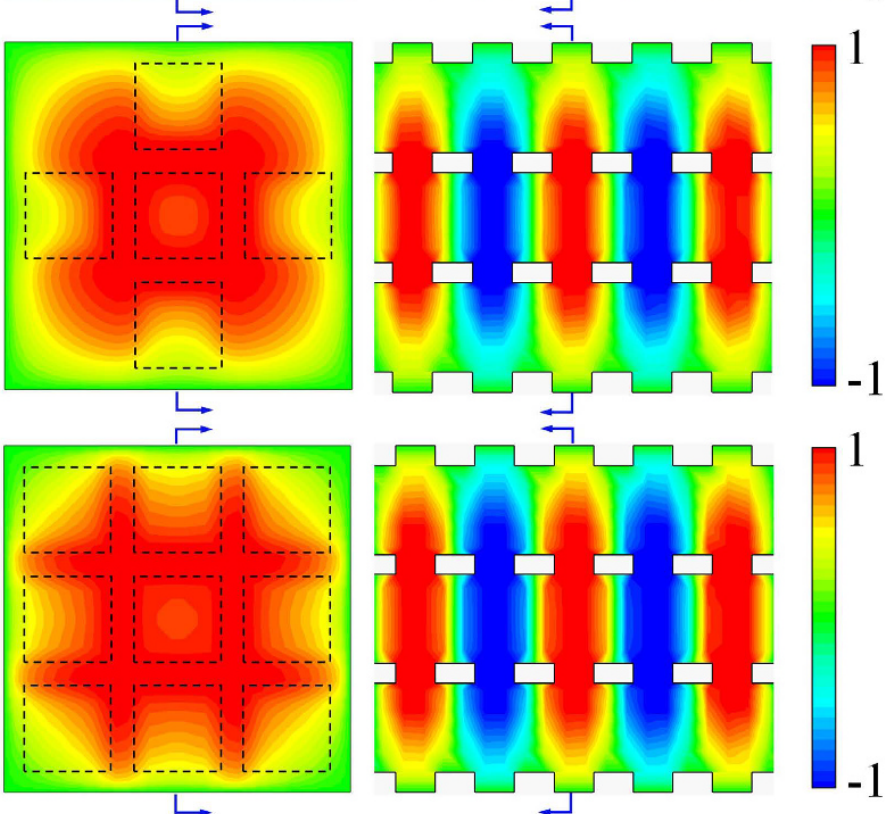

Figure 2. The simulated cross-sectional (left) and longitudinal (right) $E_{z}$ field distributions of the waves in structures with different holes. (a) 1-hole structure. (b) 3-hole structure. (c) 5-hole structure. (d) 9-hole structure (3DHA).

Moreover, by comparing the contour map of the field in Fig. 2, it is shown that the coupling has enhanced the field intensity. Next, we have undertaken a comparison of amplitude of the longitudinal electric field $\left(E_{z}\right)$ among the modes in the 3DHA, 5-hole, 3-hole, and 1-hole structures under PEC boundary condition by a 3D finite-difference time-domain (FDTD) simulation ${ }^{40}$. From the comparison result shown in Fig. 4, it can be found that the field intensities of the mode in the 3DHA are much stronger than those in the rest structures. As we know, in the beam-wave interaction, the intensity of $E_{z}$ at the e-beam location determines the interaction efficiency and radiation intensity. Thus, it can be expected that the multiple beam-MSP wave interaction in the 3DHA structure may be quite favorable.

Multi-beam interaction in 3DHA. We now analyze the multi-beam excitation and interaction with such mode in 3DHA system. First of all, when the longitudinal direct-current (DC) e-beam is injected in the modulation area, the fundamental mode of the structure will be excited. The synchronization and interaction occurs when the phase velocity of the mode matches the velocity of the e-beam. During the interaction, the DC e-beams exchange energy with the mode so the velocity and density of the DC e-beams will be modulated and the DC e-beams be bunched. Figure 3 illustrates the mechanism of the interaction. The synchronization condition is fulfilled $k_{z}=\omega / v_{z}$, where $v_{z}$ is the beam velocity. The intersection between the e-beam line and the dispersion 


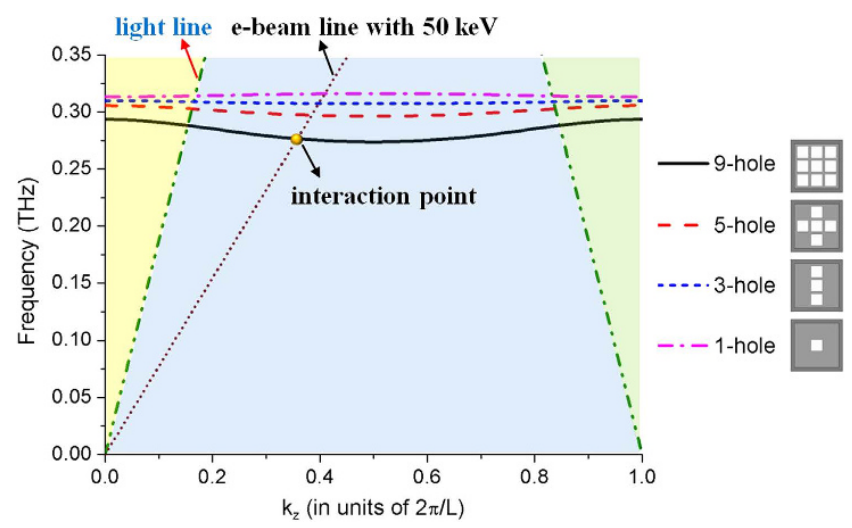

Figure 3. Mechanism of the interaction in the 3DHA system and dispersions of the fundamental modes in different hole-array structures calculated by CST Microwave Studio.

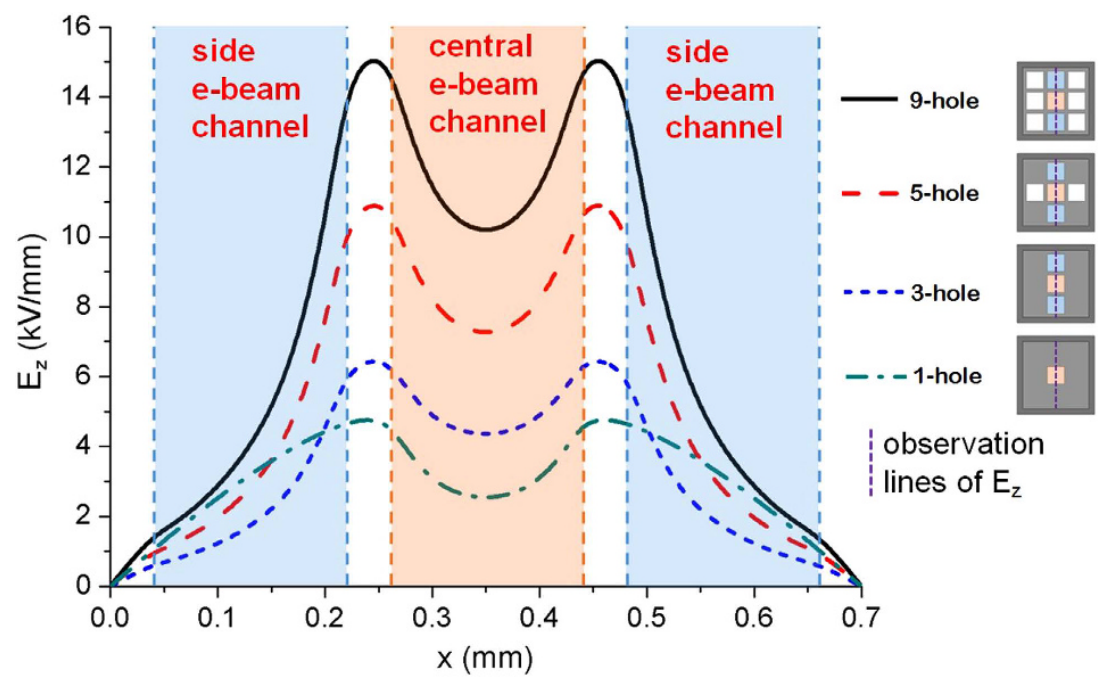

Figure 4. Comparison of amplitude of electric field $E z$ among the coupling modes in the 3DHA, 5-hole, 3-hole, and 1-hole structures.

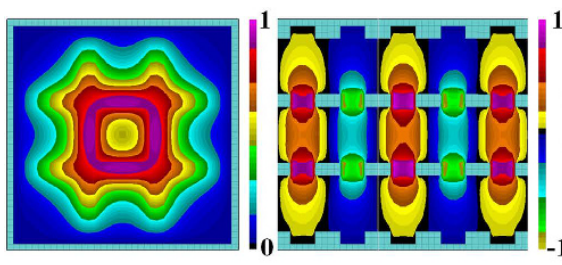

(a)

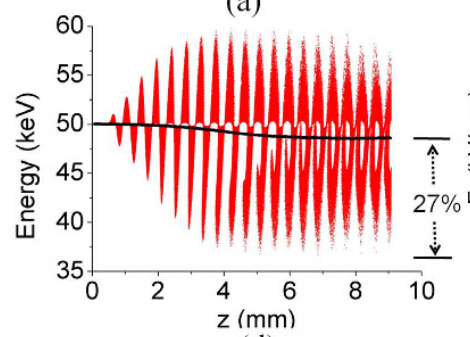

(d)

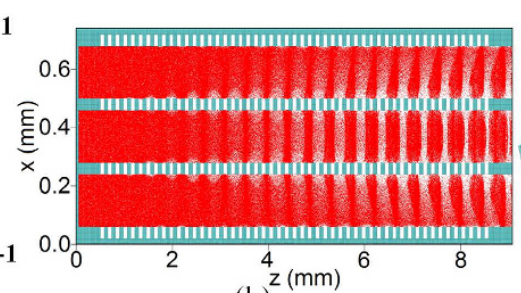

(b)

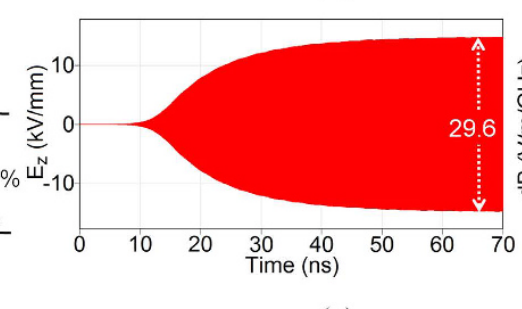

(e)

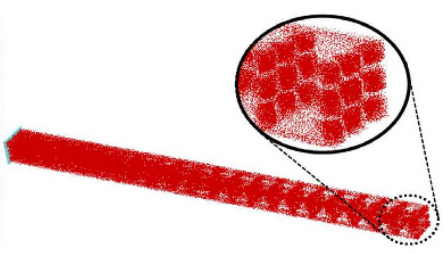

(c)

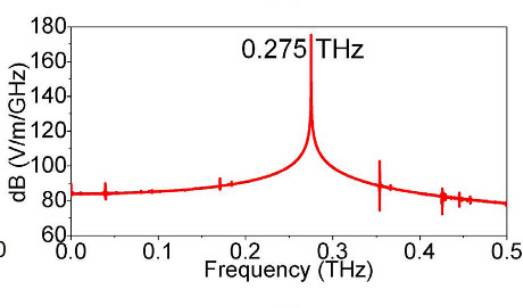

(f)

Figure 5. Simulation results of multiple beam-MSP wave interaction in the 3DHA. (a) The contour maps of electrical field $E_{z}$ (normalized) at the cross section (left) and longitudinal section (right). The phase space of the multi-beams in profile (b) and 3-D view (c). (d) The energy distribution and average energy of the multi-beams. $(\mathbf{e}, \mathbf{f})$ The time domain waveform of $E_{z}$ and its frequency spectrum. 


\begin{tabular}{|l|c|c|}
\hline Parameter & Symbol & Value \\
\hline Period of 3DHA & $L$ & $160 \mu \mathrm{m}$ \\
\hline Number of Periods & $N$ & 50 \\
\hline Thickness of hole-layer & $a$ & $80 \mu \mathrm{m}$ \\
\hline Side length of larger hole & $w$ & $700 \mu \mathrm{m}$ \\
\hline Side length of smaller hole & $g$ & $180 \mu \mathrm{m}$ \\
\hline Distance between adjacent smaller holes & $d$ & $40 \mu \mathrm{m}$ \\
\hline Distance between larger hole and side smaller hole & $h$ & $40 \mu \mathrm{m}$ \\
\hline Electron kinetic energy & $T$ & $50 \mathrm{keV}$ \\
\hline Operating current density & $J$ & $6 \mathrm{~A} / \mathrm{cm}^{2}$ \\
\hline Axial guiding magnetic field & $B$ & $0.5 \mathrm{~T}$ \\
\hline
\end{tabular}

Table 1. Parameters of the system.

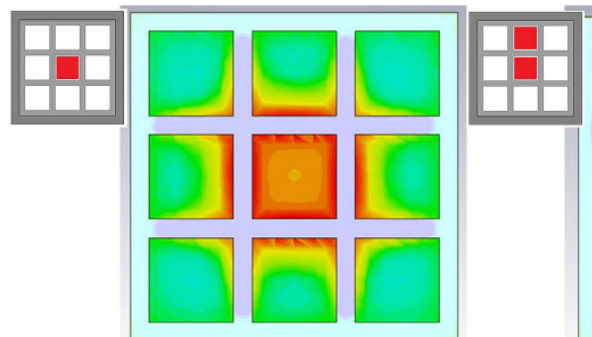

(a)

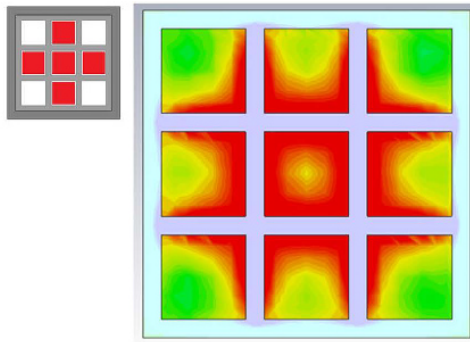

(d)

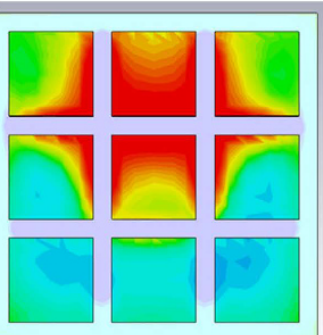

(b)
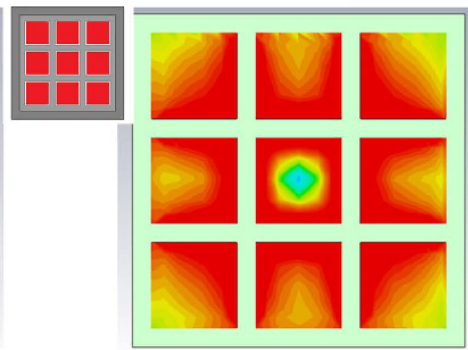

(e)
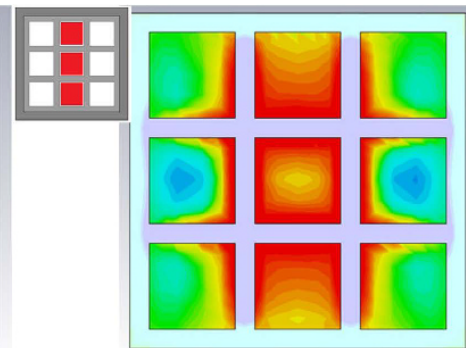

(c)

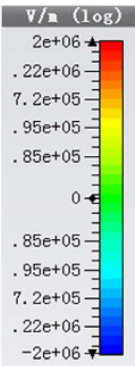

$-2 e+067$

Figure 6. The field intensity distribution of the space charge wave with different numbers of e-beams. (a) the one-beam interaction. (b) The 2-beam interaction. (c) The 3-beam interaction. (d) the 5-beam interaction. (e) the 9-beam interaction).

curve of the mode in the 3DHA (the interaction point shown in Fig. 3) is just fulfilled the condition. Therefore the interaction frequency is determined by the dimensional parameters and the beam voltage. The parameters of the system are listed in the Table 1.

A $3 \mathrm{D}$ simulation has been performed with a fully EM particle-in-cell (PIC) code CHIPIC ${ }^{41}$ based on the FDTD method applying the parameters listed in Table 1 to simulate the interaction. The $3 \times 3$ multi-beam array pass through the structure to stimulate the EM mode. In Fig. 5(a), the special coupling mode is clearly excited and the field distribution is almost the same as the analysis of the eigenmode without the e-beams in Fig. 2(d). Figure 5(b,c) demonstrate the phase space of the multi-beams in profile and 3-D view, respectively. During the interaction, the DC multi-beams are found to synchronize and interact efficiently with the coupling mode and then are gradually modulated and well bunched. It can be observed that in Fig. 5(d) the e-beam exchange the energy with the mode efficiently so that the modulation depth which is directly related to the interaction efficiency can reach near $30 \%$. Figure 5(e) shows that the amplitude of $E_{z}$ in the structure can reach $14.8 \mathrm{kV} / \mathrm{mm}$. Both modulation depth and amplitude of $E_{z}$ are relative high values for VEDs in the THz region. Figure 5(f) presents the frequency spectrum, fast Fourier transformed from the time domain waveform (Fig. 5(e)). The interaction frequency $0.275 \mathrm{THz}$ agrees well with the dispersion relation portrayed in Fig. 3. More important, the operating current density of the interaction is only $6 \mathrm{~A} / \mathrm{cm}^{2}$ which is a fairly low value for VEDs in the THz region.

Next, we have studied the coupling among the e-beams. The simulation results are shown as below. The Fig. 6 is the field intensity of the space charge wave field of the e-beam. The colors describe the field intensity, the deeper the larger. It can be found that for one e-beam as shown in Fig. 6(a), the e-beam just interact with the mode of its own hole. Therefore, the space charge field is not very strong and concentrates around the hole. It should be 


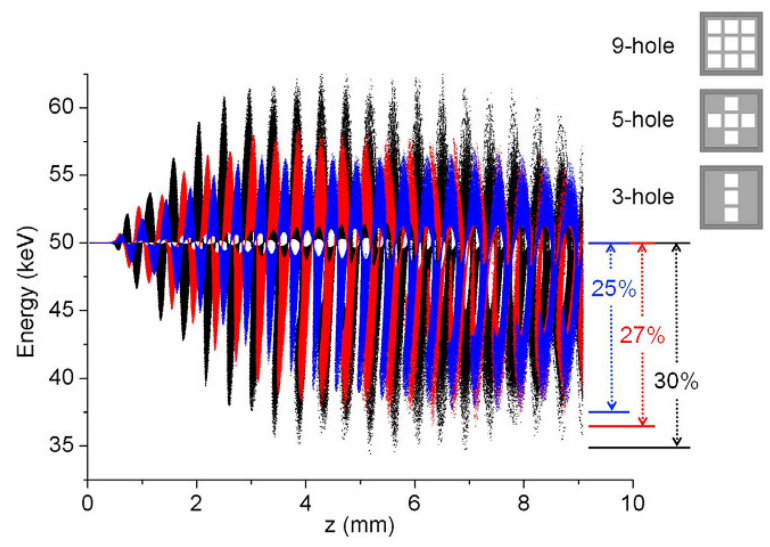

Figure 7. Comparison of modulation depth of the multi-beams among the interactions in 3DHA, 5-hole and 3-hole structures with the same current density of the multi-beams around $0.28 \mathrm{THz}$ working frequency.

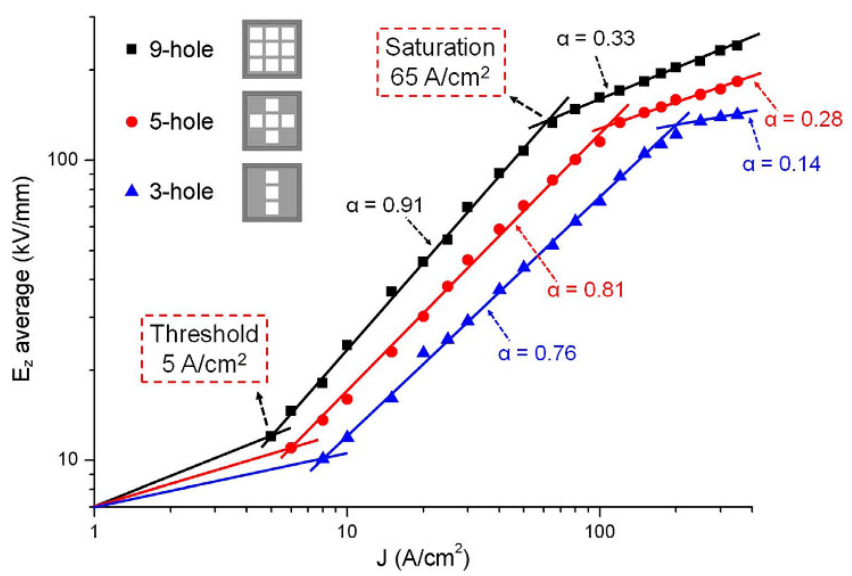

Figure 8. Radiation intensity versus beam current density in the 3DHA (9-beam), 5-hole (5-beam) and 3-hole (3-beam) structures simulated by CHIPIC. Fits were made of the form $y=A x^{\alpha}$.

noted that one period of this structure is composed of two layers, the one is with a large hole and the other is with $3 \times 3$ holes array. Therefore, in the 2-beam interaction shown in Fig. 6(b), when the beams pass the larger hole-layer, they meet with each other face to face. Then the space charge waves of both e-beams couple with each other through the hole so that the field intensity has been improved. It can be observed that at the border between the two beams, the field has enhanced significantly. With the number of e-beam increased, the coupling intensity is enhanced. For the 3-beam and 5-beam interaction, the Fig. 6(c,d) demonstrated that hence the center beam couple with beside beams the field intensity at the edge becomes larger and the size of the strong field location also has been enlarged. As a result, as shown in Fig. 6(e), due to the strong coupling among the 9-beam, the field intensity become much higher and such field covers larger region.

Moreover, the space charge wave in the e-beam directly corresponds to the modulation and efficiency. Thus, in order to further illustrate the role of coupling in the multi-beams-3DHA interaction, different number of holes and e-beams interaction have been applied in the simulation as shown in Fig. 7. It is clear that with the beam-number decreasing the modulation depth is becoming smaller. Such results show that the coupling among e-beams can enhance the interaction efficiency to improve the modulation depth.

It is known that the coherent radiation requires high current density (generally $>30 \mathrm{~A} / \mathrm{cm}^{2}$ ) for the VEDs in THz frequency band ${ }^{11}$, which is a key factor limiting developments of the e-beam-driven $\mathrm{THz}$ oscillator-sources. In this radiation system, the starting current density is considerably low. In a 50 -period 3DHA structure, fixing the beam energy at $50 \mathrm{keV}$, we sweep the beam current density. The optimized simulation results are shown as black squares in Fig. 7 . The region between the threshold and saturation points is the linear growing region where the radiation intensity from the interaction linearly increases with the current density. The starting current density is only $5 \mathrm{~A} / \mathrm{cm}^{2}$, which is a fairly low value for $\mathrm{THz}$ radiation sources, and the saturation point is about $65 \mathrm{~A} / \mathrm{cm}^{2}$. Besides, we also obtained the optimized radiation intensities as a function of the beam current density in the 5-hole (5-beam) and 3-hole (3-beam) cases, which are respectively illustrated as red circle and blue triangle in 


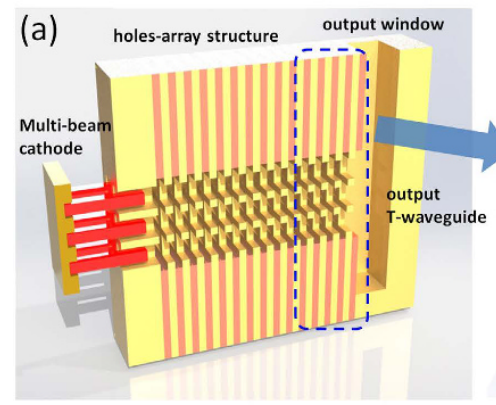

(b)

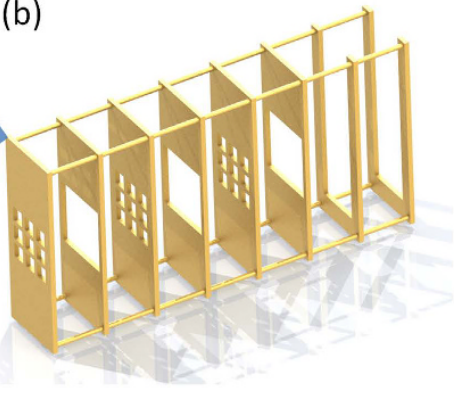

(c)

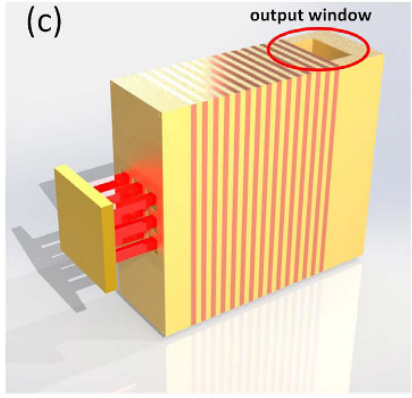

(d)
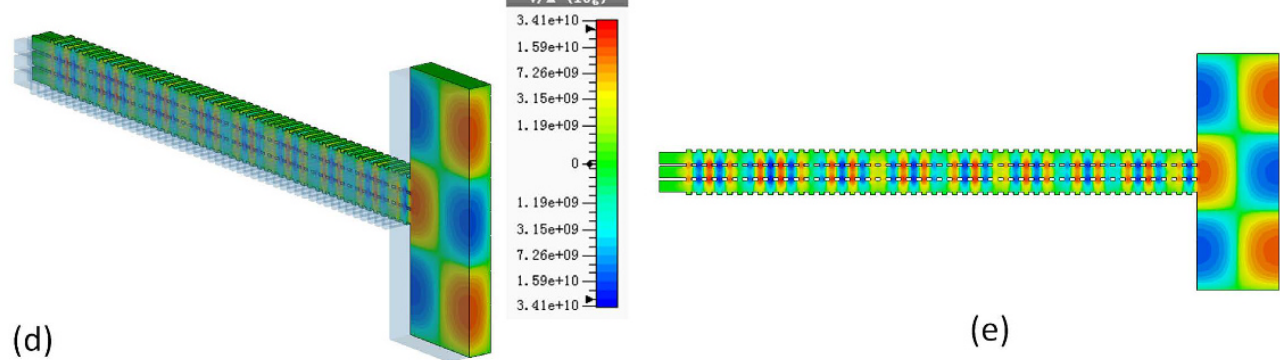

(e)

Figure 9. The skech map of the design of the whole system. (a) the 3-D view of the cross-section of the whole schematic structure of the system. (b) Sketch of assembling (c) (a) the 3-D view of the the whole schematic structure. (d) The 3D contour map of the output field. (e) The 2D contour map of the output field.

Fig. 8. It can be found that the threshold and saturation current density decrease with the number of e-beams increases, which could be considered as the coupling among the multi-beams could enhance the interaction coupling impedance so that the starting current can be reduced.

Next, the output structure has been studied. As shown in Fig. 9(a,b) and Fig. 9(c), for this radiation system the T-coupler waveguide has been applied to output the radiation. The output window is just a standard waveguide port which can connect with an antenna to emit the $\mathrm{THz}$ beam. The contour map of the output field is illustrated in Fig. 9(d) and (e). It is clearly that the fundamental mode $\mathrm{TE}_{01}$ could be observed.

Moreover, for this structure, the simpler fabrication procedure could be easier than traditional gratings. In general, grating, bi-grating and so on are used the entirely machined technique and the parameters of such structures are always very small at $\mathrm{THz}$, so the microfabrication techniques such as lithographie, galvanoformung und abformung process (LIGA) and deep reactive ion etching (DRIE) have been used. For this structure, it can be made of stacked multiple planar sub-wavelength holes layers. Thus, we can fabricate the holes layers separately and assemble them together to construct the whole structure.

In summary, generation of coherent terahertz radiation from an interaction between multi-electron beam with the coupling mode in a plasmonic crystal-like structure (three-dimensional hole-array structure) which is made of multiple stacked layers with $3 \times 3$ subwavelength holes array is proposed in this paper. The results show that such multi-beam interaction mechanism can enhance the modulation depth and reduce the current density. Due to multi-beam working, this mechanism can generate high power $\mathrm{THz}$ radiation. Moreover, this plasmonic crystal-like structure could provide larger size with more number of holes so that more than $3 \times 3$ multi-beam array can be applied in this radiation source. At last, this concept of multi-beam excitation within three-dimensional hole-array structure could provide a promising way to develop the compact $\mathrm{THz}$ radiation sources with middle or high power.

\section{References}

1. Siegel, P. H. Terahertz technology. IEEE Trans. Microwave Theory Tech. 50, 910-928 (2002).

2. Tounochi, M. Cutting-edge terahertz technology. Nat. Photonics 1, 97-105 (2007).

3. Liu, S. et al. Surface polariton Cherenkov light radiation source. Phys. Rev. Lett. 109, 153902 (2012).

4. Fülöp, J. A., Pálfalvi, L. Almási, G. \& Hebling, J. Design of high-energy terahertz sources based on optical rectification. Opt. Express 18, 12311-12327 (2010).

5. Avestisyan, Y. et al. Terahertz generation by optical rectification in lithium niobate crystal using a shadow mask. Opt. Express 20, 25752-25757 (2012).

6. Nagai, M. et al. Broadband and high power terahertz pulse generation beyond excitation bandwidth limitation via $\chi^{(2)}$ cascaded processes in $\mathrm{LiNbO}_{3}$. Opt. Express 17, 11543-11549 (2009).

7. Samoska, L. A. An overview of solid-state integrated circuit amplifiers in the submillimeter-wave and THz regime. IEEE Trans. THz Sci. Technol. 1, 9-24 (2011).

8. Chattopadhyay, G. Technology, capabilities, and performance of low power terahertz sources. IEEE Trans. THz Sci. Technol. 1, 33-53 (2011).

9. Deal, W. et al. THz monolithic integrated circuits using InP high electron mobility transistors. IEEE Trans. THz Sci. Technol. 1, 25-32 (2011).

10. Parker, R. K., Abrams, R. H. Jr., Danly, B. G. \& Levush. B. Vacuum electronics. IEEE Trans. Microwave Theory Tech. 50, $835-845$ (2002). 
11. Booske, J. H. Plasma physics and related challenges of millimeter-wave-to-terahertz and high power microwave generation. Phys. Plasma 15, 055502 (2008).

12. Booske, J. H. et al. Vacuum electronic high power terahertz sources. IEEE Trans. THz Sci. Technol. 1, 54-75 (2011).

13. Zhang, Y., Zhou, Y. Dong, L. \& Liu, S. Coherent terahertz radiation from high-harmonic component of modulated free-electron beam in a tapered two-asymmetric grating structure. Appl. Phys. Lett. 101, 123503 (2012).

14. Joye, C. D., Calame, J. P., Cook, A. M. \& Garven, M. High-power copper gratings for a sheet-beam traveling-wave amplifier at G-band. IEEE Trans. Electron Devices 60, 506-509 (2013).

15. Komandin, G. A. et al. BWO generators for terahertz dielectric measurements. IEEE Trans. THz Sci. Technol. 3, 440-444 (2013).

16. Zhou, Y., Zhang, Y., Jiang, G. \& Wu, Z. Coherent terahertz radiation generated from a square-shaped free-electron beam passing through multiple stacked layers with sub-wavelength holes. J. Phys. D: Appl. Phys. 48, 345102 (2015).

17. Kong, L. B., Huang, C. P., Du, C. H., Liu, P. K. \& Yin, X. G. Enhancing spoof surface-plasmons with gradient metasurfaces. Scientific Reports. 5, 8772 (2015).

18. Zhao, T. et al. Coherent and tunable terahertz radiation from graphene surface plasmon polarirons excited by cyclotron electron beam. Scientific Reports. 5, 16059 (2015).

19. Mizuno K. \& Ono, S. Comment on 'Travelling wave oscillations in the optical region: A theoretical examination'. J. Appl. Phys. 46, 1849 (1975).

20. Boyd, R., Dehn, R. A., Hickey, J. S. \& Mihran, T. G. The multiple-beam klystron. IRE Trans. Electron Devices, 9, 247-252 (1962).

21. Gelvich, E. A. et al. The new generation of high-power multiple-beam klystron. IEEE Trans. Microwave Theory Tech. 41, 15-19 (1993).

22. Piestrup, M. A. et al. Multiple beam free-electron lasers and optical klystrons. Appl. Phys. Lett. 39, 696-698 (1981).

23. Freund, H. P., Dougls, D. \& O'Shea, P. G. Multiple beam free-electron lasers. Nucl. Instrum. Methods Phys. Res. A 507, 373-377 (2003).

24. Shin, Y. M. et al. Superradiant terahertz Smith-purcell radiation from surface plasmon excited by counterstreaming electron beams. Appl. Phys. Lett. 90, $031502(2007)$

25. Shin, Y. M., So, J. K., Won, K. H., Srivastava, A. \& Park, G. S. Evanescent tunneling of an effective surface plasmon excited by convection electrons. Phys. Rev. Lett. 99, 147402 (2007).

26. Zhang, Y., Zhou, Y., Dong, L. \& Liu, S. Terahertz free electron superradiation from mimicking surface plasmons-two electron beams interaction within a 3-mirror quasi-optical cavity. Appl. Phys. Lett. 102, 211104 (2013).

27. Zhang Y. \& Dong, L. Enhanced coherent terahertz Smith-Purcell superradiation excited by two electron-beams. Opt. Express 20, 22627-22635 (2012).

28. Zhang, Y., Zhou, Y. \& Dong, L. THz radiation from two electron-beams interaction within a bi-grating and a sub-wavelength holes array composite sandwich structure. Opt. Express 21, 21951-21960 (2013).

29. Yan, S., Su, W., Xu, A. \& Wang, Y. Analysis of multi-beam folded waveguide traveling-wave tube for terahertz radiation. J. Electromaget. Wave. 29, 436-447 (2015).

30. Gong, Y. et al. A 140-GHz two-beam overmoded folded-waveguide traveling-wave tuve. IEEE Trans. Plasma Sci. 39, 847-851 (2011).

31. Liu, G., Wang, J., Xu, G., Yang, L. \& Luo, Y. 1.2 THz backward wave radiation generated by double bunched sheet beams. Proceedings of IEEE International Vacuum Electronics Conference (IEEE, 2015), pp. 1-2.

32. Shin, Y. M. Superimposed coherent terahertz wave radiation from mono-energetically bunched multi-beam. Phys. Plasma 19, 063115 (2012).

33. Gee A. \& Shin, Y. M. Gain analysis of higher-order-mode amplification in a dielectric-implanted multi-beam traveling wave structure. Phys. Plasma 20, 073106 (2013).

34. Yan, S., Su, W., Xu, A. \& Wang, Y. Analysis of higher order mode multi-beam folded waveguide TWT. Proceedings of IEEE International Vacuum Electronics Conference (IEEE, 2015), pp. 1-2.

35. Nusinovich, G. S., Cooke, S. J., Botton, M. \& Levush, B. Wave coupling in sheet- and multiple-beam traveling-wave tubes. Phys. Plasma 16, 063102 (2009).

36. Bouillard, J. S., Vilain, S., Dickson, W., Wurtz G. A. \& Zayats A. V. Broadband and broadangle SPP antennas based on plasmonic crystals with linear chirp. Scientific Reports. 2, 829 (2012).

37. Lin, T. R., Lin C. H. \& Hsu J. C. Strong optomechanical interaction in hybrid plasmonic-photonic crystal nanocavities with surface acoustic waves. Scientific Reports. 5, 13782 (2015).

38. Truong, T. T. et al. Nanopost plasmonic crystals. Nanotechnology 20, 434011 (2009).

39. CST Corp. CST MWS Tutorials. Available at: https://www.cst.com/.

40. Yee, K. S. Numerical solution of initial boundary value problems involving Maxwell's equations in isotropic media. IEEE Trans. Antennas Propag. 14, 302-307 (1966).

41. Zhou, J., Liu, D., Liao, C. \& Li, Z. CHIPIC: an efficient code for electromagnetic PIC modeling and simulation. IEEE Trans. Plasma Sci. 37, 2002-2011 (2009).

\section{Acknowledgements}

This work is supported by National Natural Science Foundation of China under Contract Nos 61270011, 91438118, and 61501094, National Key Basic Research Program of China under Contract No. 2014CB339806, Program for New Century Excellent Talents in University of Ministry of Education of China (Grant No. NECT_13_0100) and Sichuan Youth Science and Technology Foundation No. 2014JQ0013.

\section{Author Contributions}

Y.Z. presented the idea, guided the research work and wrote the manuscript, Y.Z., G.Y. and G.J. carried out the computer simulations, Y.Z. and Z.Y. discussed the research and revised the manuscript.

\section{Additional Information}

Competing financial interests: The authors declare no competing financial interests.

How to cite this article: Zhang, Y. et al. Coherent Terahertz Radiation from Multiple Electron Beams Excitation within a Plasmonic Crystal-like structure. Sci. Rep. 7, 41116; doi: 10.1038/srep41116 (2017).

Publisher's note: Springer Nature remains neutral with regard to jurisdictional claims in published maps and institutional affiliations. 
(c) (i) This work is licensed under a Creative Commons Attribution 4.0 International License. The images or other third party material in this article are included in the article's Creative Commons license, unless indicated otherwise in the credit line; if the material is not included under the Creative Commons license, users will need to obtain permission from the license holder to reproduce the material. To view a copy of this license, visit http://creativecommons.org/licenses/by/4.0/

(C) The Author(s) 2017 